\title{
Knowledge and Readiness of Community Health Volunteers to Lead Sensitization of Communities on COVID-19 Vaccination in Kenya: A Crosssectional Study
}

Joachim 0. Osur ( $\square$ Joachim.osur@amref.org )

Amref Health Africa https://orcid.org/0000-0003-2566-1553

Evelyne OF Muinga

Amref Health Africa

\section{Edward Ireri}

Amref International University

Jane Carter

Amref Health Africa

Shiphrah Kuria

Amref Health Africa

Salim Hussein

ministry of health

Research

Keywords: COVID-19 vaccine, vaccine hesitancy, community health volunteers

Posted Date: August 2nd, 2021

DOl: https://doi.org/10.21203/rs.3.rs-732068/v1

License: (c) (i) This work is licensed under a Creative Commons Attribution 4.0 International License. Read Full License 


\section{Abstract}

Background

Vaccination is anticipated to bring the COVID-19 pandemic to an ultimate end. Community health volunteers (CHVs) are the link between communities and the formal health system and are therefore a vital factor in successful vaccine rollout in Kenya. However, the ability of CHVs to lead community sensitization on COVID-19 vaccination was uncertain. The aim of this study was to assess the knowledge of CHVs on COVID-19 vaccination, and determine if their knowledge is adequate to lead sensitization of communities in the national COVID-19 vaccination programme.

Methods

This was a mixed methods study comprising a cross-sectional survey and key informant interviews. Quantitative data were collected from $413 \mathrm{CHVs}$ in four counties of Kenya through telephone interviews; 12 key informants were also interviewed through telephone interviews. SPSS version 25.0 and R script programming were utilised to analyse quantitative data. Qualitative data were analyzed using MAXQDA software.

Results

Of $413 \mathrm{CHVs}$ surveyed, $82.3 \%$ felt inadequately informed to engage with communities on COVID-19 vaccination. There was a significant difference in the level of knowledge between $\mathrm{CHVs}$ in urban compared to rural counties $(P=0.0005)$. The level of knowledge was also higher among more educated $\mathrm{CHVs}$ compared to less educated $\mathrm{CHVs}(\mathrm{OR}=3.04,95 \% \mathrm{Cl}: 2.47-3.61 ; p<$ 0.001). Knowledge on COVID-19 vaccine was higher in CHVs who had previously received accredited training on COVID-19 $(\mathrm{OR}=1.86,95 \% \mathrm{Cl}: 1.28-2.45 ; p<0.001)$ and this had a significant influence on CHV's willingness to be vaccinated. CHVs with higher levels of knowledge were more likely to express readiness to engage with communities on COVID-19 vaccination than those with lesser knowledge $(P=0.0001)$.

Conclusion

CHVs in four counties of Kenya did not have adequate knowledge on COVID-19 vaccination which is a major constraint to their work of mobilising communities to accept COVID-19 vaccination.

\section{Background}

Coronavirus disease 2019 (COVID-19) is a contagious viral disease caused by a novel coronavirus, severe acute respiratory syndrome coronavirus 2 (SARS-CoV-2). COVID-19 has caused major health and economic impacts globally since first reported to the World Health Organization (WHO) on December 31, 2019. WHO declared the outbreak a Public Health Emergency of International Concern on 30 January 2020, and a pandemic on 11 March, 2020. The number of confirmed COVID-19 cases worldwide as of July 21, 2021, were 183,560,151, with a total of 3,978,581 confirmed deaths. Confirmed cases of COVID-19 in Africa were 4,197,551 with 98,144 confirmed deaths ${ }^{15}$; in Kenya, the number of reported cases were 185,868 with 8,675 deaths ${ }^{15}$.

Several measures have been put in place to prevent the spread of COVID-19 including hand washing, wearing of masks, social distancing, case identification, isolation of infected people, tracing of contacts, and quarantining of contacts. However, despite substantial investments in interventions to prevent the spread of COVID-19, and in diagnosis and treatment, vaccination presents the ultimate strategy to combat the COVID-19 pandemic. Private companies and governments have worked together since the outbreak began to develop effective vaccines ${ }^{14}$. There are currently more than 50 COVID-19 vaccine candidates undergoing trials, with at least six already being rolled out in more than a dozen countries: Pfizer-BioNTech, Moderna, AstraZeneca, Sputnik $V$, Sinopharm and Bharat Biotech vaccines. Efficacy has not been confirmed in all but the Pfizer-BioNTech and Moderna vaccines have an efficacy of over $90 \% 4,8$; the AstraZeneca vaccine has an efficacy of $63.09 \%$ against SARS-CoV-2 symptomatic infections $^{15}$. The Sputnik $V$ vaccine has a reported efficacy of $91.6 \%$ against COVID-19 ${ }^{4}$ 
Nevertheless, as the world moves towards rolling out mass COVID-19 vaccination, it is likely to be hindered by vaccine hesitancy, opposition and conspiracy theories. Vaccine hesitancy and opposition may result from several factors including low knowledge, myths and misconceptions, deliberate misinformation, and social, cultural, religious and political opposition ${ }^{5}$. Moreover, concerns about the safety and efficacy of the COVID-19 vaccine may influence uptake levels and increase vaccine hesitancy, especially given the short time to develop and trial the vaccines ${ }^{3}$.

The phenomenon of vaccine hesitancy is present in all parts of the world and is spreading fast through globalized social media $^{9}$. The situation in Africa is no different from other parts of the world. A study from the Democratic Republic of the Congo revealed that only $27.7 \%$ of healthcare workers were willing to accept the vaccine if available ${ }^{7}$. With communities becoming fatigued and frustrated by the protracted nature of the pandemic, and with more and more people abandoning basic control measures, there is a high possibility that scepticism, distrust and defiance against COVID-19 prevention measures will continue to grow. Within this increasing negative context and amid globalised conspiracy theories spreading through social media, vaccine uptake is likely to face challenges unless measures are put in place to strengthen community receptiveness. Having community health volunteers (CHVs)s lead COVID-19 vaccine community engagement will play a vital role in creating confidence in COVID-19 vaccination, increasing acceptance, and reducing vaccine hesitancy and opposition among community members.

The knowledge level of community health volunteers on the importance of COVID-19 vaccination is critical for enhancing the effectiveness of vaccination programmes. Studies have shown that inadequate knowledge levels and perceptions of people on the importance of vaccination, which is related to misinformation spread on social media and anti-vaccination campaigns, influences their decision to accept COVID-19 vaccination. According to Facciolà et al. ${ }^{2}$, physicians and health workers were the main sources of information leading to vaccine opposition and hesitancy among community members. When healthcare workers and community health volunteers have the right information about the benefits and importance of vaccination, they are therefore likely to influence the public to accept vaccination and lower hesitancy levels. Community health volunteers have direct contact with community members and any information they provide is likely to be readily adopted by the community (Facciolà et al., 2019).

This study aimed at assessing the knowledge, attitudes, values and practices of community health volunteers (CHVs) on COVID19 vaccination in Kenya, and identifying knowledge gaps, conspiracy theories, negative beliefs and readiness to accept vaccination.

\section{Methodology}

\section{Study design, sample size, and participants}

Cross-sectional surveys and key informant interviews were conducted in four counties in Kenya (Mombasa, Nairobi, Kajiado, and Trans Nzoia), from March to April, 2021. Purposive sampling was used to select the study counties based on their level of urbanisation, with Mombasa and Nairobi counties representing urban counties, and Kajiado and Trans Nzoia representing rural counties (nomadic and agrarian communities respectively). The list of CHVs registered with the Ministry of Health in the selected counties formed the sampling frame, and simple random sampling was used to select the study sample. 413 community health volunteers were randomly chosen proportionate to the population size of each region: Nairobi 209 (51\%), Mombasa 84 (21\%); Kajiado 54 (13\%); and Trans Nzoia 66 (15\%).

The eligible study population were $\mathrm{CHV}$ s registered to practise, those attached to a link health facility, and those who had worked as a CHV for at least one year. CHVs who had retired or had worked for less than one year were excluded from the study. Key informant interviews (KII) were conducted with county directors of health, county community strategy focal persons, sub-county community strategy focal persons, community health extension workers (CHEW) or community health assistants (CHA), a representative community health volunteer, and community leaders from each selected county.

\section{Survey tools and data collection}


The survey questionnaires and key informant guide were derived, adapted and conceptualised from the WHO SAGE vaccine hesitancy matrix (WHO, 2011). The questions focused on testing knowledge of CHVs on COVID-19 vaccines; and were set in English, one of Kenya's official languages. The questionnaire had two sections: part one consisted of the socio-demographic characteristics of the participants, and part two contained the knowledge questions. The key informant guide was developed from the gaps identified after quantitative data analysis.

In line with protocols for combating the spread of COVID-19 disease, telephone interviews were adopted to collect quantitative data while virtual meetings were held to collect qualitative data. Research assistants were trained on the study and study tools, and how to collect data through telephone interviews. Interviews were conducted after receiving verbal consent to participate from the respondents. KIls were conducted through virtual meetings after consent for participation and recording was obtained. Interviews were recorded for quality purposes.

\section{Data management and analysis}

The collected study data were cleaned, coded and entered into SPSS version 25 for analysis. Quantitative variables were organised and summarised using frequencies and percentages. For objectives 1 and 2, quantitative data were explored using descriptive and Chi-square statistics. Significant categorical variables were further analysed using binary logistics regression to determine odds ratios. Qualitative data from the key informant interviews were transcribed, cleaned, coded and analysed thematically.

\section{Results}

\section{Socio-demographic characteristics of the CHVs}

Table 1 shows the socio-demographic characteristics of the total sample of $413 \mathrm{CHVs}$ who consented to participate in the study, comprising 108 (26.2\%) men, 301 (72.9\%) women, and 4 (1\%) who indicated 'others'. Most respondents were 35 years of age and above (64.4\%); the age distributions were 18 - 24 years (7.5\%), $25-35$ years (28.1\%), and above 35 years (64.4\%). Religion distribution was Catholics 98 (23.7\%), Protestants 233 (56.4\%), Islam 38 (9.2\%), and others 44 (10.7\%). The majority had worked for more than 5 years $(62.2 \%)$ as $\mathrm{CHVs}$. The education level distribution was none $(0.7 \%)$, primary level $(28.1 \%)$, secondary level (49.5\%), and others (21.5\%). CHVs who had attended approved COVID-19 training by the Ministry of Health were $74.6 \% ; 5.8 \%$ were not sure if they had attended, and 19.6\% had not attended any COVID-19 approved training; $86.2 \%$ of the CHVs had taken part in educating the community about COVID-19, but $13.8 \%$ had not been involved in any COVID-19 education of the community. The main source of income for CHVs was non-formal employment (51.1\%), while $43.1 \%$ of the CHVs obtained their income from $\mathrm{CHV}$ work, and $5.8 \%$ obtained their income from other formal work. 
Socio-demographic characteristics of community health volunteers

\begin{tabular}{|c|c|}
\hline Variables & Frequencies \\
\hline Sex: & $108(26.2 \%)$ \\
\hline Male & $301(72.9 \%)$ \\
\hline Female & $4(1 \%)$ \\
\hline \multicolumn{2}{|l|}{ Others } \\
\hline Age (years): & $31(7.5 \%)$ \\
\hline $18-24$ & $116(28.1 \%)$ \\
\hline $25-35$ & $266(64.4 \%)$ \\
\hline \multicolumn{2}{|l|}{35 and above } \\
\hline Religion: & $98(23.7 \%)$ \\
\hline Catholic & $233(56.4 \%)$ \\
\hline Protestant & $38(9.2 \%)$ \\
\hline Islam & $44(10.7 \%)$ \\
\hline \multicolumn{2}{|l|}{ Others } \\
\hline Education level: & $3(0.7 \%)$ \\
\hline None & $116(28.1 \%)$ \\
\hline Primary & $205(49.5 \%)$ \\
\hline Secondary & $89(21.5 \%)$ \\
\hline \multicolumn{2}{|l|}{ Others } \\
\hline Years of service as a CHV: & $71(17.2 \%)$ \\
\hline Less than 3 years & $85(20.6 \%)$ \\
\hline $3-5$ years & $257(62.2 \%)$ \\
\hline \multicolumn{2}{|l|}{ Above 5 years } \\
\hline Attended $\mathrm{MoH}$ approved training: & $308(74.6 \%)$ \\
\hline Yes & $81(19.6 \%)$ \\
\hline No & $24(5.8 \%)$ \\
\hline \multicolumn{2}{|l|}{ Not sure } \\
\hline Involvement in educating the community on COVID-19: & $356(86.2 \%)$ \\
\hline Yes & $57(13.8 \%)$ \\
\hline \multicolumn{2}{|l|}{ No } \\
\hline Main source of income: & $178(43.1 \%)$ \\
\hline CHV work & $24(5.8 \%)$ \\
\hline Other formal employment & $211(51.1 \%)$ \\
\hline Other non-formal employment & \\
\hline
\end{tabular}


Table 2 summarizes CHV's knowledge on COVID-19 vaccines. The majority of CHVs (92.7\%) had attended a course on COVID-19 vaccines. However, most CHVs (93.5\%) did not have access to COVID-19 vaccine information such as information, education, and communication (IEC) materials which could be used to disseminate accurate messages about COVID-19 vaccines to communities. However, $66.3 \%$ of $\mathrm{CHVs}$ had persons (CHEW/CHA) designated to them whom they could consult on COVID-19 vaccination matters. Most CHVs did not know how the vaccine works (51.3\%), 37.5\% had an idea but were not confident to explain this to the community, and $11.2 \%$ felt they had sufficient knowledge on how COVID-19 vaccines work to be able to explain to community members; $39.2 \%$ CHVs felt they were not adequately informed about COVID-19 vaccines to sensitise their families or community members; $87.3 \%$ CHVs were not aware of what COVID-19 vaccines contain. The majority of $\mathrm{CHVs}$ (77\%) felt that after receiving COVID-19 vaccination, people should continue to observe COVID-19 prevention and control measures; $3.6 \%$ felt that it was not necessary to continue to observe COVID-19 protocols, while $19.4 \%$ did not know what should be done after people receive COVID-19 vaccine. Some CHVs indicated that they were still in doubt about the effectiveness of the COVID19 vaccine in preventing infections (34.6\%), while 35.6\% did not know about the effectiveness of the COVID-19 vaccine; only $29.8 \%$ felt that the effectiveness of COVID-19 vaccines in preventing infection is quite high in vaccines that have been approved. On people who should be vaccinated, the following categories of people were proposed to be vaccinated first: school-going children $(3.4 \%)$, children under five years $(2.3 \%)$, adolescents $(0.9 \%)$, youths $(1.1 \%)$, elderly people $(13.3 \%)$, adults $(3.3 \%)$, all people $233(36.3 \%)$, health care workers $(19 \%)$, CHWs $(9.8 \%)$, and others $(10.5 \%)$. 


\begin{tabular}{|c|c|}
\hline Variables & Frequencies \\
\hline Attended a course on COVID-19 vaccination: & $30(7.3 \%)$ \\
\hline Yes & $383(92.7 \%)$ \\
\hline \multicolumn{2}{|l|}{ No } \\
\hline \multirow{2}{*}{$\begin{array}{l}\text { Access to COVID-19 vaccination materials: } \\
\text { Yes No }\end{array}$} & $27(6.5 \%)$ \\
\hline & $386(93.5 \%)$ \\
\hline \multirow{2}{*}{$\begin{array}{l}\text { Have a designated person with knowledge on COVID-19 vaccination whom you can consult when you have a } \\
\text { question on COVID-19 vaccination: }\end{array}$} & $274(66.3 \%)$ \\
\hline & $139(33.7 \%)$ \\
\hline \multicolumn{2}{|l|}{ 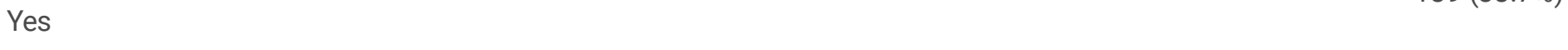 } \\
\hline \multicolumn{2}{|l|}{ No } \\
\hline Concerning how COVID-19 vaccines work to prevent infection: & $46(11.2 \%)$ \\
\hline I can explain to the community how the vaccines work & $\begin{array}{l}155(37.5 \\
\%)\end{array}$ \\
\hline \multicolumn{2}{|l|}{ I have an idea but I am not confident to explain to the community how the vaccines work } \\
\hline \multicolumn{2}{|l|}{ I do not know how the vaccines work } \\
\hline \multirow[t]{2}{*}{$\begin{array}{l}\text { The extent to which CHVs feel adequately informed about COVID-19 vaccine to sensitizs their } \\
\text { family/community members: }\end{array}$} & $162(39.2 \%)$ \\
\hline & $178(43.1 \%)$ \\
\hline Not at all & $60(14.5 \%)$ \\
\hline Small extent & $12(2.9 \%)$ \\
\hline Average extent & $1(0.2 \%)$ \\
\hline \multicolumn{2}{|l|}{ Large extent } \\
\hline \multicolumn{2}{|l|}{ Very large extent } \\
\hline Do you know what the COVID-19 vaccines contain? & $50(12.1 \%)$ \\
\hline Yes & $363(87.9 \%)$ \\
\hline \multicolumn{2}{|l|}{ No } \\
\hline What should people who have received the COVID-19 vaccine do? & $15(3.6 \%)$ \\
\hline Do not have to wear masks, social distance or wash hands frequently to prevent COVID-19 & $318(77 \%)$ \\
\hline Still have to wear masks, social distance, and wash hands frequently to prevent COVID-19 & $80(19.4 \%)$ \\
\hline \multicolumn{2}{|l|}{ I do not know } \\
\hline The effectiveness of COVID-19 vaccines in preventing infection: & $123(29.8 \%)$ \\
\hline Is quite high in vaccines that have been approved & $143(34.6 \%)$ \\
\hline $\begin{array}{l}\text { Is still in doubt and will only be known once the COVID-19 vaccines that have been approved are used for } \\
\text { some time }\end{array}$ & $147(35.6 \%)$ \\
\hline I do not know & \\
\hline
\end{tabular}




\begin{tabular}{|lc|}
\hline Variables & Frequencies \\
\hline Who should be vaccinated? & $22(3.4 \%)$ \\
School-going children & $15(2.3 \%)$ \\
Children under five years & $6(0.9 \%)$ \\
Adolescents & $7(1.1 \%)$ \\
Youth & $85(13.3 \%)$ \\
Elderly people & $21(3.3 \%)$ \\
Adults & $233(36.3 \%)$ \\
All people & $122(19 \%)$ \\
Health care workers & $63(9.8 \%)$ \\
CHWs & $67(10.5 \%)$ \\
Others & \\
\hline
\end{tabular}

The results from the qualitative survey showed that the knowledge level of CHVs and healthcare workers about COVID-19 vaccination was very low, as low as 10\%. Most of the CHVs had been trained on COVID-19 disease but had not been trained on the COVID-19 vaccine. Moreover, the health workers who were supposed to train the CHVs on COVID-19 vaccination were reported not to have undergone any training on COVID-19 vaccination. Two respondents from different counties reported:

"However, for the vaccine not much effort has been put there so their knowledge is 10\%, they are like the common person. Their supervisors (CHAs/CHEWs) are also not trained on the same." KII

Most of them do not have knowledge of the vaccine, I think they should be trained to equip their communities with information on the vaccine". And for the issue of COVID, concerning COVID-19 vaccine the knowledge is low, I can say it is very low because there have not been forums for the CHVs discussing the issues of COVID-19 vaccine. So I can say the knowledge gap is maybe, it I can give it percentage, I can say it is $10 \%$ because we have not educated them concerning the vaccine. $~ \mathrm{KII}$

\section{Sources of information on COVID-19 vaccination}

Figure 1 shows the main sources of information on COVID-19 vaccination.

Television and radio (59.5\%) were the major sources of information for CHVs on COVID-19 vaccination. The next common sources of information were social media (21.2\%), community members (10.7\%), information, education and communication (IEC) materials given out by the Ministry of Health (6\%), and $2.5 \%$ from other sources.

"On the vaccine, their knowledge is very low because they have not been trained on the vaccine. They are getting the information about the vaccine from the TV and other social media." KII

Binary logistics regression identified two main factors affecting participants' readiness to engage with communities: county of origin, level of education, and previous experience in educating communities on COVID-19. There was a significant difference in level of knowledge between $\mathrm{CHVs}$ in urban compared to rural counties $(P=0.0005)$. Level of knowledge was also higher among more educated $\mathrm{CHVs}$ and those who had previously received accredited training on COVID-19 (OR = 3.04, 95\% Cl: 2.47-3.61; $p<$ 0.001). CHVs with higher levels of knowledge were more likely to express readiness to engage with communities on vaccination than those with lower knowledge $(P=0.0001)$. Knowledge concerning how the COVID-19 vaccines work to prevent infection significantly influenced the willingness of $\mathrm{CHVs}$ to be vaccinated (adjOR $=2.58,95 \% \mathrm{Cl}: 1.94-3.22 ; p<0.001)$. Knowledge of the effectiveness of COVID-19 vaccines in preventing infection (adjOR $=3.26,95 \% \mathrm{Cl}$ : $2.34-4.17 ; p<0.001)$ also significantly influenced vaccine uptake intention. The extent to which the CHVs felt that they were well-informed about COVID-19 vaccines influenced their ability to sensitize their families and community members (OR $=1.86,95 \% \mathrm{Cl}: 1.28-2.45 ; p<0.001)$. 
From the key informant interviews on the training needs required to equip CHVs with proper knowledge on COVID-19 vaccination included educating $\mathrm{CHV}$ s on the content of the COVID-19 vaccines because many CHVs did not know what the vaccines contain, how they work, how they are administered, the possible side effects of the vaccines, and who should be vaccinated.

"...CHVs need written information on the vaccine, what kind of vaccine it is, how it is administered, the side effects and overall information on it. And they need also to understand who does not qualify so that they can clear that with the community. Having written material acts as a reference point whenever a CHV is required to give information on the vaccine. It's a source document." $\sim \mathrm{KII}$

The key informants reported that the safety concerns and importance of the COVID-19 vaccine need to be incorporated into the training of CHVs, so they are able to demystify myths and misinformation circulated through social media. Some of the myths and misinformation which were reported to be spread through social media on safety concerns of COVID-19 vaccine included: COVID-19 vaccine causes blood clots; the vaccine causes death; the vaccine causes impotence in young men and infertility in women of reproductive age.

"...we need to put a lot of effort in enlightening CHVs on the safety of this vaccine, safety of this vaccine is very important. ... and then there are so many rumours and misconceptions on the vaccine on social media. So they need to be cleared, especially those that are on social media; they are affecting the understanding of the importance of a vaccine to a CHV. So this needs to be cleared, so that they can separate fact and fiction." KII

"The other one they say that when it gets to the body, it causes the blood to stop, blood will not flow. We are hearing in America there was something to do with clot so they don't want to. So they are saying that we are not going to be experimental. We are not going to volunteer ourselves to be jab only to die. Those are the fears and the misconceptions." KII

"They say that it will harm his/her life, because it destroys the blood. That when you are vaccinated the vaccine goes to the blood. " KII

"Personal beliefs I have heard which are weird and which we must confront is that people are saying that these vaccines, if you are vaccinated then women will be impotent. They will not have, I mean they will not bear children. Things like that. We are hearing very weird way of reasoning about this vaccine. That if it is a younger man it will interfere with some DNA and the young man he will also be impotent he will not sire children. Those are the things we are confronting in the villages." KII

The key informants indicated that the types of COVID-19 vaccine and period of protection after vaccination requires to be included in the CHVs' training on COVID-19 vaccination. The training contents should also include demonstrations of the different types of vaccine so the CHVs are able to differentiate them.

"... how long is the vaccine preventing them from getting COVID-19, the people who believe that if you get the vaccine you no longer get COVID-19, who are the people that should be vaccinated, the different types of vaccines that are there, you know they also read and they know there are several types, they should be told, where they are administered, they have also seen the other vaccines how they look like, how different is that from this other one because they are the people who will pass the information to the common citizen and they should tell them it is a vaccine like the other vaccines. When one is vaccinated how long does it take for one to be protected? You know the issue of protection after getting the vaccine. " KII

"The trainers can use demonstrations to show how the vaccine looks like, where the vaccine is injected into the body... and the type of the vaccines available." KII

\section{Discussion}

Vaccination is one of the most effective techniques for preventing spread of infectious viral diseases globally. Vaccination has been proved effective for control of polio, rubella and measles, and was solely responsible for the eradication of smallpox ${ }^{1}$. Therefore, there was urgency by scientists to develop new vaccines to protect populations from COVID-196. 
In this study, $87.3 \%$ of CHVs had no knowledge of COVID-19 vaccines, how they work, what the vaccines contain, and the effectiveness of the vaccines. According to Facciolà et al. ${ }^{2}$, the knowledge level of community health workers on COVID-19 vaccines is critical as it influences their acceptance of a vaccine, as well as motivating them to mobilise communities to accept vaccines. The qualitative results of this study indicated that CHVs had very low knowledge of COVID-19 vaccines; only about $10 \%$ of CHVs said they were knowledgeable about COVID-19 vaccines. This lack of knowledge would affect CHV's ability to educate communities about COVID-19 vaccines, and may result in ineffective programming.

According to the Theory of Planned Behaviour, people make informed, reasoned, and logical decisions to take part in a specific behaviour by examining the information accessible to them ${ }^{10}$. They will then perform the behaviour depending on the perceived usefulness of the information they have. The results of our study revealed that CHVs in four counties of Kenya felt inadequately informed to educate community members on COVID-19 vaccination. This low knowledge level is concurrent with the emergency introduction of vaccines in the country as was reported by many respondents from the key informant interviews. The low knowledge of $\mathrm{CHVs}$ on COVID-19 vaccines could also be explained by the high level of misinformation from social media and other forms of media as these were indicated as major sources of information on COVID-19 vaccines. CHVs who had been previously engaged in training communities on COVID-19 vaccination had a higher knowledge of COVID-19 vaccines compared to those who had not engaged in educating communities. CHVs with higher education levels had greater knowledge about COVID-19 vaccines compared to those with lower education levels; this was associated with access to information on COVID-19 vaccines $\mathrm{CHVs}$ who had concerns on how the vaccines work had greater hesitancy towards uptake of vaccination.

Television and radio were reported to be the most used channels of communication by CHVs to access information on COVID-19 vaccination. Others reported that social media such as WhatsApp and Facebook were being used to propagate misinformation about COVID-19 vaccines. This is an unfortunate result of lack of Ministry of Health efforts to rapidly avail the right information to the public about COVID-19 vaccination; without available authoritative information, people opt to search for information about the vaccine from social media.

The county of origin of the CHVs had a significant association with the level of knowledge of COVID-19 vaccination. A study to determine COVID-19 hesitancy from various countries indicated that preference for the vaccine varied depending on rural-urban residence and regions. This might be caused by the knowledge level of the population on COVID-19 vaccines, their safety and effectiveness. Lack of correct information about COVID-19 vaccines was stated to be the major cause of COVID-19 vaccine hesitancy in sub-Saharan Africa and globally ${ }^{11,12}$. Moreover, our findings indicated that CHVs lacked knowledge of the contents of COVID-19 vaccines, a reason the CHVs felt inadequately informed to educate the community on COVID-19 vaccination.

\section{Conclusion}

CHVs were found to have inadequate knowledge to be able to effectively lead community sensitisation on COVID-19 vaccination: $50 \%$ did not feel informed at all and $37 \%$ felt informed only to a small extent. CHVs had mixed knowledge on vaccine eligibility, need for preventive measures after vaccination and need for vaccination by COVID-19 survivors. Major differences in readiness to engage with communities on vaccination were dependent on county of origin and especially on whether CHVs has previously participated in sensitising communities on COVID-19. CHVs with low levels of knowledge reported they were not confident enough to carry out COVID-19 vaccine sensitisation. However, with the safety concerns, importance of the vaccine, demystifying of myths and misinformation, and the types and period of protection after vaccination incorporated into the training of $\mathrm{CHVs}$, the confidence of the $\mathrm{CHVs}$ to carry out community vaccination education could be increased.

\section{RECOMMENDATION}

1. The findings of this study suggest the importance of training community health workers on COVID-19 and its vaccines to reduce hesitancy, and funds for training must accompany vaccination porgrammes.

2. The study findings recommend supporting CHVs in Kenya to enhance COVID-19 vaccine rollout and vaccination campaigns, through training, provision of equipment, deployment of $\mathrm{CHVs}$ and monitoring their performance. 
3. There should be enhanced provision of information and educational materials, and engagement with $\mathrm{CHVs}$ to increase trust in Ministry of Health messaging around the vaccine.

\section{Abbreviations}

$\begin{array}{ll}\mathrm{CHV} & \text { Community Health Volunteers } \\ \mathrm{KII} & \text { Key Informant Interviews } \\ \mathrm{MoH} & \text { Ministry of Health } \\ \mathrm{WHO} & \text { World Health Organization }\end{array}$

\section{Declarations}

\section{Ethics approval and consent to participate}

The research protocol was approved by the Ethics and Scientific Review Committee of Amref Health Africa. The administrative permit to conduct the research was given by the National Commission for Science, Technology and Innovation (NACOSTI). Informed consent was sought from all study participants to ensure voluntary participation. Study information collected in hard copy was kept confidential National Commission for Science, Technology and Innovation under lock and key, while recordings from key informant interviews were password secured until the final data were analysed and then the recordings and the hard copies were destroyed. Codes were used instead of participants' names on the questionnaire to ensure anonymity. All Research Assistants and any other staff with access to data signed Confidentiality Agreements before the study started.

\section{Consent for publication}

All the authors gave their consent for the manuscript to be published.

\section{Availability of data and materials}

The data included in this manuscript were as collected from the respondents and analysed without alteration for any reason.

\section{Competing interests}

The authors declare that there was no conflict of interest in publication of the manuscript.

\section{Funding}

The research was funded by Amref Health Africa.

\section{Authors' contributions}

Prof. Joachim Osur: conceptualisation, protocol co-writer, oversight of data collection, analysis, co-writer of the draft manuscript, review and editing of the manuscript, and publishing.

Evelyne Muinga: conceptualisation, designing and writing of the research protocol, data collection, oversight of data management, validation and verification of the data, writing and editing of the manuscript, and publishing.

Edward Ireri: methodology, review of the protocol, statistical analysis and validation of the results.

Dr Jane Carter: Funding acquisition, review and editing of the research protocol, and review and editing of the manuscript.

Dr Shiphrah Kuria: review and editing of the protocol, review and editing of the manuscript.

Dr Salim A. Hussein: review of the protocol and review of the manuscript. 


\section{ACKNOWLEDGEMENTS}

We wish to thank Amref Health Africa for their support during the performance of this study. Specifically, we would like to thank Prof. Joachim Osur, Vice-Chancellor, Amref International University, for providing timely feedback and quality control over the entire study. We appreciate the contributions made by every team member (Evelyne Muinga, Edward Ireri, Dr Shiphrah, and Dr Salim Hussein) in supporting the entire research process. Special thanks to Dr. Carter for mobilising funds for the study, and for severally reviewing the draft manuscript. he team wishes to express their gratitude to Rehema Chengo, onitoring and Evaluation Officer, Amref Health Africa, Headquaeters, for technical support during the data collection and dissemination process. Finally, we would like to thank all staff and cooperating partners at national, county, and community levels who aided us during the entire process and made the research possible.

\section{References}

1. Dubé E. Addressing vaccine hesitancy: the crucial role of healthcare providers. Clinical Microbiology and Infection. 2017 May 1;23(5):279-80.

2. Facciolà A, Visalli G, Orlando A, Bertuccio MP, Spataro P, Squeri R, Picerno I, Di Pietro A. Vaccine hesitancy: An overview on parents' opinions about vaccination and possible reasons of vaccine refusal. Journal of public health research. 2019 Mar $11 ; 8(1)$.

3. Fadda M, Albanese E, Suggs LS. When a COVID-19 vaccine is ready, will we all be ready for it?.

4. Logunov DY, Dolzhikova IV, Shcheblyakov DV, Tukhvatulin Al, Zubkova OV, Dzharullaeva AS, Kovyrshina AV, Lubenets NL, Grousova DM, Erokhova AS, Botikov AG. Safety and efficacy of an rAd26 and rAd5 vector-based heterologous prime-boost COVID-19 vaccine: an interim analysis of a randomised controlled phase 3 trial in Russia. The Lancet. 2021 Feb 20;397(10275):671-81.

5. MacDonald NE. Vaccine hesitancy: Definition, scope and determinants. Vaccine. 2015 Aug 14;33(34):4161-4.

6. McAteer J, Yildirim I, Chahroudi A. The VACCINES Act: deciphering vaccine hesitancy in the time of COVID-19. Clinical Infectious Diseases. 2020 Jul 28;71(15):703-5.

7. Nzaji MK, Ngombe LK, Mwamba GN, Ndala DB, Miema JM, Lungoyo CL, Mwimba BL, Bene AC, Musenga EM. Acceptability of vaccination against COVID-19 among healthcare workers in the Democratic Republic of the Congo. Pragmatic and observational research. 2020;11:103.

8. Polack FP, Thomas SJ, Kitchin N, Absalon J, Gurtman A, Lockhart S, Perez JL, Marc GP, Moreira ED, Zerbini C, Bailey R. Safety and efficacy of the BNT162b2 mRNA Covid-19 vaccine. New England Journal of Medicine. 2020 Dec 10.

9. Puri N, Coomes EA, Haghbayan H, Gunaratne K. Social media and vaccine hesitancy: new updates for the era of COVID-19 and globalized infectious diseases. Human vaccines \& immunotherapeutics. 2020 Nov 1;16(11):2586-93.

10. Ryan S, Carr A. Applying the biopsychosocial model to the management of rheumatic disease. InRheumatology 2010 Jan 1 (pp. 63-75). Churchill Livingstone.

11. Sallam M. COVID-19 vaccine hesitancy worldwide: a concise systematic review of vaccine acceptance rates. Vaccines. 2021 Feb;9(2):160.

12. Tulloch O, Roldan de Jong T, Bardosh K. Data Synthesis: COVID-19 Vaccine Perceptions in Sub-Saharan Africa: Social and Behavioural Science Data, March 2020-April 2021.

13. WHO. Report of the SAGE Working Group on Vaccine Hesitancy. 2014 October 1 Accessed at https://www.who.int/immunization/sage/meetings/2014/october/1_Report_WORKING_GROUP_vaccine_hesitancy_final.pdf

14. WHO . COVID-19 Case Definition.2020 September 21 Retrieved from https://covid-19.who.int/

15. WHO , COVID-19 Dashboard. 2021 July 21Retrieved from https://covid19.who.int/table

\section{Figures}

Page $12 / 13$ 


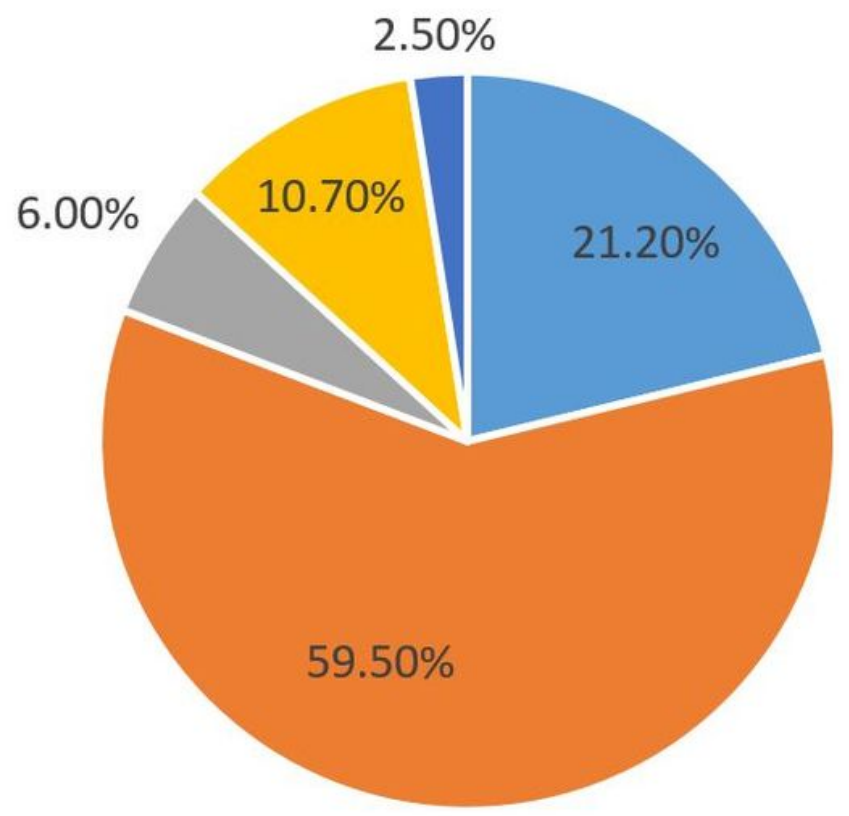

- social media

- radio and TV

- IEC materials from $\mathrm{MoH}$

= communit members

- other sources

Figure 1

Sources of information on COVID-19 vaccination 